\title{
The Influence of the Frontal Surface Area and Swim Velocity Variation in Front Crawl Active Drag
}

\author{
JORGE E. MORAIS ${ }^{1,2}$, ROSS H. SANDERS ${ }^{3}$, CHRISTOPHER PAPIC ${ }^{3}$, TIAGO M. BARBOSA ${ }^{1,2}$, \\ and DANIEL A. MARINHO ${ }^{2,4}$ \\ ${ }^{1}$ Instituto Politécnico de Bragança, Bragança, PORTUGAL; ${ }^{2}$ Research Centre in Sports, Health and Human Development \\ (CIDESD), University of Beira Interior, Covilhã, PORTUGAL; ${ }^{3}$ Exercise and Sport Science, Faculty of Medicine and Health, \\ University of Sydney, Sydney, AUSTRALIA; and ${ }^{4}$ University of Beira Interior, Covilhã, PORTUGAL
}

\begin{abstract}
MORAIS, J. E., R. H. SANDERS, C. PAPIC, T. M. BARBOSA, and D. A. MARINHO. The Influence of the Frontal Surface Area and Swim Velocity Variation in Front Crawl Active Drag. Med. Sci. Sports Exerc., Vol. 52, No. 11, pp. 2357-2364, 2020. Purpose: The aims of this study were to 1) compare active drag $\left(D_{\mathrm{a}}\right)$ calculation between a single land-based measurement of frontal surface area (FSA) and in-water FSA measures obtained at key events of the arm pull (1, right upper-limb catch; 2, right upper-limb insweep; 3, right upper-limb exit and left upper-limb catch; 4, left upper-limb insweep; and 5, left upper-limb exit and right upper-limb catch) at front crawl swimming, and 2) compare mechanical power variables computed based on these two approaches. Methods: Seventeen swimmers (11, male; 6, female; $16.15 \pm 0.94 \mathrm{yr}$ old) were recruited. The FSA was measured based on two approaches: (i) nonvariation, that is, assuming a constant value, and (ii) variation, that is, calculated in each key event of the front crawl swim. Active drag based on a nonvariation of the FSA was measured using the Velocity Perturbation method. Active drag based on a variation approach was measured in each key event of the front crawl according to the law of linear motion. Paired $t$-test $(P \leq 0.05)$, simple linear regression models, and Bland-Altman plots between assessment methods (variation vs nonvariation) were computed. Results: The FSA (variation) was higher than when assuming a nonvariation $(0.1110 \pm 0.010$ vs $0.0968 \pm 0.010 \mathrm{~m}^{2}, \Delta=15.69 \%, t=4.40, P<0.001, d=0.95$ ). Active drag (variation) was also significantly higher than when assuming a nonvariation $(88.44 \pm 25.92$ vs $75.41 \pm 15.11 \mathrm{~N}, \Delta=16.09 \%, t=3.66, P=0.002, d=0.61)$. Conclusions: Besides the FSA, swim velocity also changes during the front crawl arm pull. The variation of both variables had a significant effect on the active drag measurement and consequently on mechanical power and total power input variables. Key-words: SWIMMING, DRAG, DYNAMIC VERSUS STATIC MEASUREMENTS
\end{abstract}

$\mathrm{T}$ he hydrodynamics of swimming is a challenging scientific field for researchers, for both data collection and analysis, because of the complex behavior of water. To complete a given swimming distance in minimum time, swimmers may increase their forward velocity by increasing propulsion and/or decreasing hydrodynamic drag (1). Propulsion is produced by upper- and lower-limb actions such as arm stroking and leg kicking (2). Hydrodynamic drag is a force acting opposite to the relative motion of any object moving with

Address for correspondence: Jorge E. Morais, Ph.D., Department of Sport Sciences, Instituto Politécnico de Bragança, Campus Sta. Apolónia, Apartado 1101, 5301-856 Bragança, Portugal; E-mail: morais.jorgestrela@gmail.com. Submitted for publication March 2020.

Accepted for publication April 2020.

0195-9131/20/5211-2357/0

MEDICINE \& SCIENCE IN SPORTS \& EXERCISE $_{\circledast}$ Copyright $(C 2020$ by the American College of Sports Medicine

DOI: $10.1249 / \mathrm{MSS} .0000000000002400$ respect to the aquatic environment (3), and it can be estimated based on Newton's law of linear motion as:

$$
D=\frac{1}{2} \rho v^{2} \times \mathrm{FSA} \times C_{D}
$$

where $D$ is the hydrodynamic drag (in newtons), $\rho$ is the density of the water (assuming $997 \mathrm{~kg} \cdot \mathrm{m}^{-3}$ ), FSA is the frontal surface area (in square meters), $v$ is the swim velocity (in meters per second), and $C_{\mathrm{D}}$ is the drag coefficient (dimensionless).

FSA (Equation 1) corresponds to the swimmer's maximal cross-sectional area in a plane transverse to the direction of forward motion (4). The FSA can be estimated based on anthropometric features such as the chest perimeter and chest sagittal diameter (5), or measured directly by numeric data in static or dynamic conditions using digital video cameras (6) and digital photogrammetry (7). FSA is usually obtained from measurements of the swimmer on land assuming a streamlined position because this is a simple and less time-consuming method than measuring FSA while swimming (5). When the swimmer is gliding through the water without arm and leg actions, the hydrodynamic resistance is termed "passive drag" (8). In that case, the FSA does not change because there are 
no dynamic movements from the upper limbs' actions (4). Hence, researchers use a constant value (i.e., nonvariation) for FSA when calculating passive drag. When swimming with arm stroking, the hydrodynamic resistance is termed "active drag" $\left(D_{\mathrm{a}}\right)(9)$. In this case, FSA is a variable that changes during the swimming stroke because of postural changes associated with arm actions, body roll, and kicking.

Nevertheless, for convenience of calculation, researchers usually assume FSA to be a constant value during free swimming and do not take into account the dynamic movements (i.e., variation) of the swimmer's body and limbs. The one study to date that did consider varying FSA in front crawl used estimated active drag measures, and such measurements are usually calculated from a mean swimming velocity (7). Indeed, swim velocity $(v)$ is another major drag determinant (Equation 1). Active drag increases whenever the swim velocity increases, but this was shown during the entire stroke cycle (10). Drag force is proportional to squared swim velocity. Thus, using a mean swim velocity in the drag calculation, instead of its instantaneous value, might lead to a measurement bias. Moreover, one may argue that this bias is even larger assuming a mean FSA over the cycle. In front crawl, there are five key events of the arm pull: 1) right upper-limb catch, 2) right upper-limb insweep, 3) right upper-limb exit and left upper-limb catch, 4) left upper-limb insweep, and 5) left upper-limb exit and right upper-limb catch $(7,11)$. At each of these key events, the FSA differs from the FSA when in the streamlined position (7), and the swim velocity is also different (12). Consequently, this will affect active drag.

In addition to adopting different postures throughout the stroke cycle, reflected in differences in FSA between the identifiable events, the postures may differ because of asymmetries in the stroke patterns of individual swimmers. During motion forward, swimmers roll their body about the longitudinal axis while performing the arm pull (13). Because the human body is not symmetrical and swimmers may not perform each arm pull (i.e., right and left upper limb) with an equivalent spatial-temporal movement pattern (14), FSA might be meaningfully different during each phase of the stroke. Therefore, it seems to be of major importance to assess the influence of FSA on active drag during those key events of the arm pull, and not only as a mean and constant value. Furthermore, there are mechanical power and total power input (i.e., energy expenditure) variables that are usually computed/estimated based on the active drag output. Such variables reflect the swimmer's power to promote displacement while swimming, with power and hence energy expenditure being of major interest for researchers and practitioners $(15,16)$.

Given that the FSA may change dynamically (i.e., variation) during the swimming stroke, there is a need to evaluate whether a single measure of FSA is adequate for obtaining estimates of active drag and mechanical power. Therefore, the aims of this study were to 1) compare $D_{\mathrm{a}}$ calculation between a single land-based measurement of FSA and in-water FSA measures obtained at key events of the arm pull at front crawl swimming, and 2) compare mechanical power variables computed based on these two approaches. It was hypothesized that 1) $D_{\mathrm{a}}$ would be different when FSA is determined dynamically (variation) than when it is single land-based measured, and 2) mechanical power variables and energy expenditure calculated based on a variation approach would be different from when measured based in a nonvariation.

\section{METHODS}

\section{Participants}

The sample comprised 17 adolescent swimmers (11, male; 6 , female; $16.15 \pm 0.94 \mathrm{yr}$ old; $66.62 \pm 7.76 \mathrm{~kg}$ of body mass; $1.73 \pm 0.08 \mathrm{~m}$ of height; $1.79 \pm 0.11 \mathrm{~m}$ of arm span), including national record holders, age-group national champions, and other swimmers involved in a talent identification program $(584.18 \pm 55.49$ and $636.33 \pm 105.60$ Fédération Internationale de Natation points at the $100-\mathrm{m}$ freestyle event in short-course meter for males and females, respectively). The swimmers had more than $5 \mathrm{yr}$ of competitive experience and trained six to seven swimming sessions per week. Parents or guardians, and the swimmers themselves signed an informed consent form. All procedures were in accordance to the Declaration of Helsinki regarding human research, and the University Ethics Board approved the research design.

Frontal surface area (nonvariation). The FSA assuming a nonvariation during the stroke cycle was computed as being the largest area in the transverse plane of the swimmer's body while in a streamlined position (4). The swimmers, while standing upright on land and simulating the hydrodynamic position, were photographed from above at a height of $2.5 \mathrm{~m}$ with a digital camera (Alpha 6000; Sony, Tokyo, Japan). The hydrodynamic position is characterized by the upper limbs being fully extended above the head, one hand above the other, fingers also extended close together, and head in neutral position (5). They wore their regular textile swimsuit and cap. On the camera-shooting field was a calibration frame with 1-m length at the height of the xiphoid process. Afterward, the FSA was measured from the swimmer's digital photograph with a specific software (Universal Desktop Ruler, AVPSoft, Pittsburgh, PA) by two expert evaluators (intraclass correlation coefficient $=0.989)$ (17).

Active drag (nonvariation). The active drag $\left(D_{\mathrm{a}}\right)$, assuming a nonvariation, and the coefficient of active drag $\left(C_{\mathrm{Da}}\right)$ were computed based on the Velocity Perturbation method (18). It is a procedure with several advantages in comparison to other techniques to measure active drag: 1) it can be used in all swimming strokes; 2) it has a better traction as far as ecological validity is concerned; 3 ) it requires an easy setup before testing; 4) it is a straightforward procedure, that is, less time consuming and more affordable than other techniques; and 5) it can be used to assess active drag in a broad range of ages and competitive levels $(19,20)$.

Swimmers performed two 25 m maximal front crawl swimming trials with a push-off start after a standardized warm-up (21). One trial performed at maximal front crawl, and the other at maximal front crawl while towing a hydrodynamic body 
(i.e., a perturbation device). This hydrodynamic body was attached to the swimmer's waist with a belt at a distance of $8 \mathrm{~m}$ (to minimize drafting effects of the perturbation device in the wake of the swimmer) (18).

The $D_{\mathrm{a}}$ was computed as:

$$
D_{\mathrm{a}}=\frac{D_{\mathrm{b}} v_{\mathrm{b}} v^{2}}{v^{3}-\mathrm{v}_{\mathrm{b}}^{3}}
$$

where $D_{\mathrm{a}}$ (in newtons) is the swimmer's active drag at maximal velocity, $D_{\mathrm{b}}$ (in newtons) is the resistance of the hydrodynamic body computed from the manufacturer's calibration of the buoy-drag characteristics and its velocity, and $v_{\mathrm{b}}$ and $v$ (in meters per second) are the swimming velocities with and without the perturbation device. The $C_{\mathrm{Da}}$ was computed as:

$$
C_{\mathrm{Da}}=\frac{2 D_{\mathrm{a}}}{\rho \times \mathrm{FSA} \times v^{2}}
$$

where $C_{\mathrm{Da}}$ is the coefficient of active drag (dimensionless), $D_{\mathrm{a}}$ is the active drag (in newtons), $\rho$ is the density of the water $\left(997 \mathrm{~kg} \cdot \mathrm{m}^{-3}\right), \mathrm{FSA}$ is the swimmer's FSA (in square meters), and $v$ is the swim velocity (in meters per second).

Kinematics and active drag variation. The following data were retrieved during the trial without the perturbation device previously described. To measure the swim velocity, a speedo-meter string (Swim speedo-meter; Swimsportec, Hildesheim, Germany) was attached to the swimmer's waist (15). The velocity of the swimmer's waist was deemed appropriate as an indication of the velocity associated with production of drag. The velocity of the waist closely reflects the motion of the swimmer's trunk segment, that is, the body segment in which the largest FSA occurs and which therefore has the largest influence on $D_{\mathrm{a}}$. An in-house built software (LabVIEW ${ }^{\circledR}$, v. 2010$)$ was used to acquire $(f=50 \mathrm{~Hz})$ and display speed-time data over each trial. Data were exported from the speedo-meter to interface by a 12-bit resolution acquisition card (USB-6008; National Instruments, Austin, TX). Then, it was imported into a signal processing software (AcqKnowledge v. 3.9.0; Biopac Systems, Santa Barbara, CA). High frequencies of the signal were removed using a Butterworth fourth-order low-pass filter (cutoff, $5 \mathrm{~Hz}$ ). The mean swim velocity $(v$, in meters per second) was then measured between the 11th and 24th meters. A video camera (Sony FDR-X3000, Tokyo, Japan) was attached to a rail on the edge of the swimming pool at a depth of $20 \mathrm{~cm}$ filming $(f=60 \mathrm{~Hz})$ the swimmers in the transverse plane. This was synchronized to the swim velocity signal to retrieve the instantaneous swim velocity in each phase of the arm pull (for further measurements). The stroke frequency (SF) was calculated as the number of cycles per unit of time, from the time it takes to complete one full cycle $(f=1 / P$, where $P$ is the period), and afterward converted to hertz (intraclass correlation coefficient $=0.990$ ). The intracyclic variations (whenever appropriate) were calculated with the coefficient of variation $(\mathrm{CV}): \mathrm{CV}=\mathrm{SD} /$ mean $\times 100$, where $\mathrm{CV}$ is the coefficient of variation and SD is $1 \mathrm{SD}$. Hence, the intracyclic variation of the swim velocity $(d v)$ was calculated as being the $\mathrm{CV}$.

The Froude efficiency $\left(\eta_{\mathrm{F}}\right.$, in percent) was calculated as:

$$
\eta_{\mathrm{F}}=\left(\frac{0.9 v}{2 \pi \times \mathrm{SF} \times l}\right) \frac{2}{\pi} \times 100
$$

where $\eta_{\mathrm{F}}$ is the Froude efficiency (in percent), $v$ is the mean swim velocity (in meters per second), SF is the stroke frequency (in hertz), and $l$ is the shoulder to hand average distance (in meters) (22). The $l$ distance was measured by digital photogrammetry (between the acromion and tip of the third finger) (17).

Frontal surface area (variation). To assess the FSA as a changing variable (FSA_variation), five distinct key events of the arm pull were considered: 1) right upper-limb catch, 2) right upper-limb insweep, 3) right upper-limb exit and left upper-limb catch, 4) left upper-limb insweep, and 5) left upper-limb exit and right upper-limb catch (7). The images for the FSA measurement in each one of such phases were obtained by the projected image of the camera toward which the swimmer was swimming (Fig. 1, panels 1-5). Afterward, each FSA was computed by digital photogrammetry as described in the anthropometrics section. Because the swimmers did not present the same duration of the stroke cycle, the stroke time was expressed in percentage of total duration $(0 \%-100 \%)$. The values during the arm pull (for each swimmer) were interpolated by means of cubic spline from which the values of FSA were calculated in every percentage point (each 5\%) of the stroke (Fig. 2, panel 1). The intracyclic variation of the FSA (dFSA) was calculated as being the CV.

The instantaneous swim velocity in each one of the five key events of the arm pull was retrieved from the velocity-time curve (Fig. 2, panel 2). The $C_{\mathrm{Da}}$ was retrieved from the drag section (measured with the velocity perturbation method). This was considered not to change as a function of swim velocity as reported elsewhere $(7,23)$. Afterward, the active

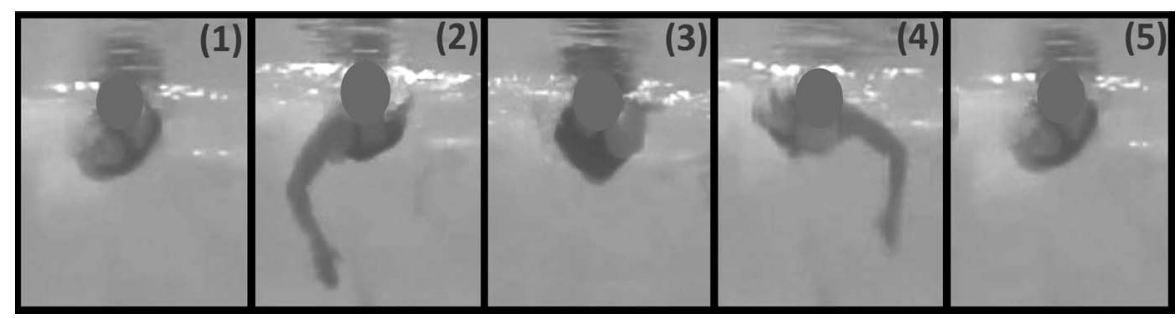

FIGURE 1-The five key events of a full stroke cycle: right upper-limb catch (panel 1), right upper-limb insweep (panel 2), right upper-limb exit and left upper-limb catch (panel 3), left upper-limb insweep (panel 4), and left upper-limb exit and right upper-limb catch (panel 5). 

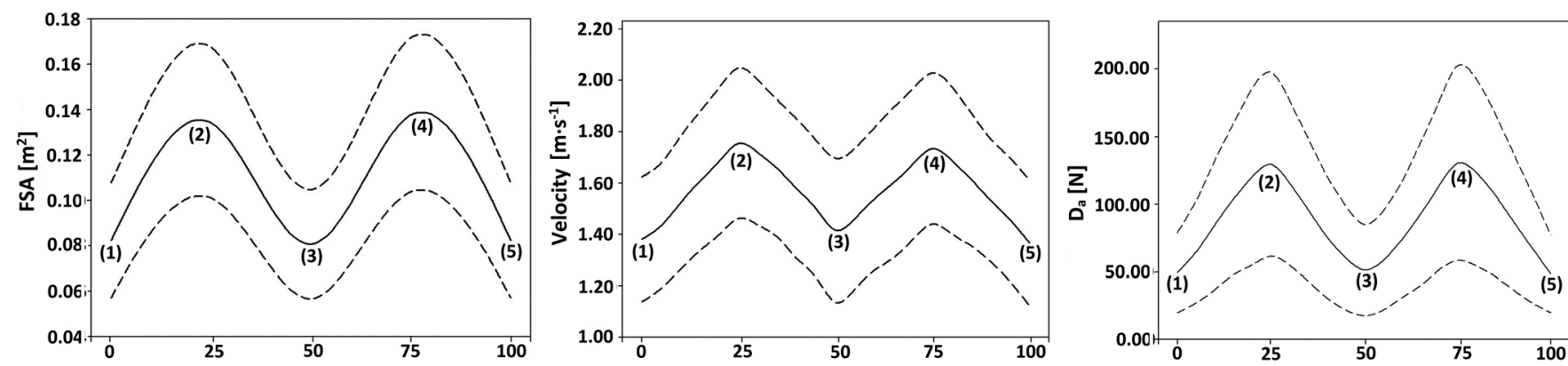

FIGURE 2-Variation during a full stroke cycle of the FSA (panel 1), swim velocity (panel 2), and $D_{\mathrm{a}}$ (active drag, panel 3) (1, right upper-limb catch; 2 , right upper-limb insweep; 3, right upper-limb exit and left upper-limb catch; 4, left upper-limb insweep; 5, left upper-limb exit and right upper-limb catch). Solid line represents the mean, dash line represents the $95 \%$ confidence interval (95CI).

drag as a changing variable ( $D_{\text {a_variation }}$; Fig. 2, panel 3$)$ was computed in every percentage point of the stroke (as the FSA) based on Equation 1, and the mean value was used for further analysis. The intracyclic variation of active $\operatorname{drag}\left(\mathrm{d} D_{\mathrm{a}}\right)$ was calculated as being the CV.

\section{Mechanical Power}

The mechanical power variables were computed twice: (i) based on the drag without variation and (ii) based on drag variation. The power to overcome $\operatorname{drag}\left(P_{\mathrm{d}}\right)$ was computed as:

$$
P_{\mathrm{d}}=D_{\mathrm{a}} v
$$

where $P_{\mathrm{d}}$ is the power to overcome drag (in watts), $D_{\mathrm{a}}$ is the swimmers' active drag at maximal velocity, and $v$ is the swim velocity (in meters per second) (18). From these data, it was possible to compute the external mechanical power $\left(P_{\text {ext }}\right)$ and the mechanical power to transfer kinetic energy to water $\left(P_{\mathrm{k}}\right)(15)$. The $P_{\text {ext }}$ was computed as:

$$
P_{\text {ext }}=\frac{P_{\mathrm{d}}}{\eta_{\mathrm{F}}}
$$

where $P_{\text {ext }}$ is the external mechanical power (in watts), $P_{\mathrm{d}}$ is the power to overcome drag (in watts), and $\eta_{\mathrm{F}}$ is the Froude efficiency (dimensionless) (24). The $P_{\mathrm{k}}$ was computed as:

$$
P_{\mathrm{k}}=P_{\text {ext }}-\mathrm{P}_{\mathrm{d}}
$$

where $P_{\mathrm{k}}$ is the mechanical power to transfer kinetic energy to water (in watts), $P_{\text {ext }}$ is the external mechanical power (in watts), and $P_{\mathrm{d}}$ is the power to overcome drag (in watts). The total power input ( $\dot{E}_{\text {tot }}$ in watts) was estimated as:

$$
\dot{E}_{t o t}=\frac{P_{\mathrm{d}}}{\eta_{\mathrm{m}} \eta_{\mathrm{F}}}
$$

where $\dot{E}_{\text {tot }}$ is the total power input (in watts), $P_{\mathrm{d}}$ is the power to overcome drag (in watts), $\eta_{\mathrm{F}}$ is the Froude efficiency (dimensionless), and $\eta_{\mathrm{m}}$ the mechanical efficiency (dimensionless) (15). It was assumed an average value of 0.2 for the $\eta_{\mathrm{m}}$ as reported elsewhere (25).

\section{Statistical Analysis}

Nonsignificant Shapiro-Wilk and Levene tests showed that the assumptions of normality and homoscedasticity, respectively, were not violated. The mean plus $1 \mathrm{SD}$ and the $95 \%$ confidence interval (95CI) were computed as descriptive statistics.

Comparative analysis between the nonvariation and variation measurements (FSA, $D_{\mathrm{a}}, P_{\mathrm{d}}, P_{\text {ext }}, P_{\mathrm{k}}$, and $\dot{E}_{\text {tot }}$ ) included the following: (i) mean data comparison, (ii) simple linear regression between values, and (iii) Bland-Altman plots (26). For the mean data comparison, the Student's $t$-test paired samples $(P \leq 0.05)$, the mean difference $(\Delta)$, and the magnitude of the effect size (Cohen $d$ ) were computed. This effect size index was interpreted as follows: (i) small if $d<0.2$, (ii) moderate if $0.8>d \geq 0.2$, and (iii) large if $d \geq 0.8$ (27). Simple linear regression models between assessment methods (variation vs nonvariation) were computed. Trendline equation, determination coefficient $\left(R^{2}\right)$, adjusted determination coefficient $\left(\mathrm{Ra}^{2}\right)$, standard error of estimation (SEE), and $95 \mathrm{CI}$ and $95 \%$ prediction intervals (95PI) were calculated. As a rule of thumb and qualitative interpretation, the relationship was defined as follows: very weak if $R^{2}<0.04$, weak if $0.04 \leq R^{2}<0.16$, moderate if $0.16 \leq R^{2}<0.49$, high if $0.49 \leq R^{2} 0.81$, and very high if $0.81 \leq R^{2}<1.0$.

The Bland-Altman analysis included the plot of the mean value assuming a nonvariation of the FSA versus assuming an FSA variation (28). It was adopted as limits of agreement a bias of \pm 1.96 SD of the difference. For qualitative assessment, it was considered that the analytical modeling data were valid and appropriate if at least $80 \%$ of the plots were within the $\pm 1.96 \mathrm{SD}$ of the difference $(95 \mathrm{CI})$.

\section{RESULTS}

Table 1 presents the descriptive statistics (mean $\pm 1 \mathrm{SD}$ ) for all variables measured. The FSA (nonvariation) had a mean value of $0.0968 \pm 0.010 \mathrm{~m}^{2}$ (Table 1). Figure 2 (panels 1-3) depicts the variation of the FSA 1), $v 2$ ), and $D_{\mathrm{a}} 3$ ) during a full stroke cycle. The FSA (variation) had a mean value of $0.1110 \pm 0.010 \mathrm{~m}^{2}$ (variation). The lowest value was verified in key event three $\left(0.0806 \pm 0.012 \mathrm{~m}^{2}\right)$ and the highest in key 


\begin{tabular}{|c|c|c|c|c|c|c|c|}
\hline & \multirow[b]{2}{*}{ Nonvariation, Mean \pm 1 SD } & \multirow[b]{2}{*}{ Variation, Mean \pm 1 SD } & \multicolumn{5}{|c|}{ Five Key Events of the Arm Pull, Mean \pm 1 SD } \\
\hline & & & 1 & 2 & 3 & 4 & 5 \\
\hline $\begin{array}{l}\text { FSA, } m^{2} \\
\text { dFSA, } m^{2}\end{array}$ & $0.0968 \pm 0.010$ & $\begin{array}{c}0.1110 \pm 0.010 \\
18.57 \pm 1.89\end{array}$ & $0.0825 \pm 0.013$ & $0.1343 \pm 0.017$ & $0.0806 \pm 0.012$ & $0.1378 \pm 0.017$ & $0.0825 \pm 0.013$ \\
\hline $\begin{array}{l}D_{\mathrm{a}}, \mathrm{N} \\
\mathrm{d} D_{\mathrm{a}}, \%\end{array}$ & $75.41 \pm 15.11$ & $\begin{array}{l}88.44 \pm 25.92 \\
32.50 \pm 3.21\end{array}$ & $49.53 \pm 15.08$ & $129.72 \pm 34.72$ & $51.32 \pm 17.20$ & $130.47 \pm 36.77$ & $48.60 \pm 14.70$ \\
\hline$v, \mathrm{~m} \cdot \mathrm{s}^{-1}$ & $1.57 \pm 0.13$ & $1.57 \pm 0.13$ & $1.38 \pm 0.12$ & $1.76 \pm 0.15$ & $1.42 \pm 0.14$ & $1.74 \pm 0.15$ & $1.37 \pm 0.12$ \\
\hline $\mathrm{d} v, \%$ & $8.60 \pm 1.44$ & $8.60 \pm 1.44$ & & & & & \\
\hline$C_{\mathrm{Da}}$, dimensionless & $0.64 \pm 0.23$ & $0.64 \pm 0.23$ & & & & & \\
\hline$\eta_{F}, \%$ & $30.10 \pm 2.26$ & $30.10 \pm 2.26$ & & & & & \\
\hline$P_{\mathrm{d}}, \mathrm{W}$ & $118.67 \pm 27.64$ & $140.03 \pm 47.68$ & & & & & \\
\hline$P_{\text {ext }}, \mathrm{W}$ & $396.90 \pm 97.25$ & $465.78 \pm 154.15$ & & & & & \\
\hline$P_{\mathrm{k}}, \mathrm{W}$ & $278.22 \pm 71.47$ & $325.74 \pm 107.98$ & & & & & \\
\hline$\dot{E}_{t o t}, \mathrm{~W}$ & $1984.50 \pm 486.27$ & $2328.88 \pm 770.74$ & & & & & \\
\hline
\end{tabular}

Five key events of the arm pull: 1) right upper-limb catch, 2) right upper-limb insweep, 3) right upper-limb exit and left upper-limb catch, 4) left upper-limb insweep, and 5) left upper-limb exit and right upper-limb catch.

event four $\left(0.1378 \pm 0.017 \mathrm{~m}^{2}\right)$, with a dFSA of $18.57 \% \pm 1.98 \%$ (Table 1; Fig. 2, panel 1).

The $v$ showed a mean value of $1.57 \pm 0.13 \mathrm{~m} \cdot \mathrm{s}^{-1}$. The lowest value occurred in key event five $\left(1.37 \pm 0.12 \mathrm{~m} \cdot \mathrm{s}^{-1}\right)$ and the highest in key event two $\left(1.76 \pm 0.15 \mathrm{~m} \cdot \mathrm{s}^{-1}\right)$, with a $\mathrm{d} v$ of $8.60 \% \pm 1.44 \%$ (Table 1; Fig. 2, panel 2). The $D_{\text {a variation }}$ showed a mean value of $88.44 \pm 25.92 \mathrm{~N}$. The lowest value was verified in key event five $(48.60 \pm 14.70 \mathrm{~N})$ and the highest in key event four $(130.47 \pm 36.77 \mathrm{~N})$, with a $\mathrm{d} D_{\text {a }}$ of $32.50 \% \pm 3.21 \%$ (Table 1; Fig. 2, panel 3).

The $t$-test comparison between the variables assessed based in a nonvariation and variation revealed a significant difference for all pairwise (Table 2). The FSA presented a relative difference of $\Delta=15.69 \%(t=4.40, P<0.001 ; d=0.95)$ and a moderate relationship $\left(R^{2}=0.22, \mathrm{Ra}^{2}=0.17, P=0.055\right)$ with an SEE of $0.013 \mathrm{~m}^{2}$. The $D_{\text {a }}$ presented a relative difference of $\Delta=16.09 \%(t=3.66, P=0.002, d=0.61)$ and a high relationship $\left(R^{2}=0.76, \mathrm{Ra}^{2}=0.75, P<0.001\right)$, with an SEE of $13.01 \mathrm{~N}$.

As for the Bland-Altman analysis, in all variables assessed, only one dot was located beyond the 95CI agreement (Fig. 3). Therefore, the Bland-Altman analysis showed that more than $80 \%$ of the plots for all variables were within the $95 \mathrm{CI}$ agreement (Fig. 3).

\section{DISCUSSION}

The aims of this study were to (i) compare the $D_{\text {a }}$ calculation between a single FSA land-based measure and FSA measures obtained at key events during the stroke cycle of front crawl swimming, and (ii) compare mechanical power variables computed based on these two approaches. The $D_{\text {a variation }}$ (and remaining variables computed based on a variation measurement) was significantly higher than the $D_{\mathrm{a}}$, supporting the hypothesis that the calculated values of such variables would differ between the variation and nonvariation approaches.

To the best of our knowledge, only one study reported the importance of the FSA variation during the front crawl swim (7). In that study, FSA was determined by digital photogrammetry and it was shown that it changed during the arm pull. However, $D_{\mathrm{a}}$ was not measured individually in each one of the five key events of the arm pull. It was measured as the mean value of the entire arm pull. Therefore, a hypothetical variation of such variable during the arm pull was not assessed. Furthermore, the $D_{\mathrm{a}}$ was estimated based on equation 1 assuming the mean value of the FSA and $v$. It was not taken into consideration that FSA and $v$ change in each key event of the arm pull (Fig. 2). Moreover, the $C_{\mathrm{Da}}$ was not measured directly but assumed to be 0.3 for all swimmers, which might lead to an assessment bias (7). This would additionally increase the measurement bias because the $C_{\mathrm{Da}}$ also changes based on shape and velocity (8). In the present study, the Velocity Perturbation method was used to measure directly the swimmers' $C_{\mathrm{Da}}$ (18). This allowed for the measurement of the $C_{\mathrm{Da}}$ of each swimmer individually because it changes based on the swimmers' shape, drag, velocity, and water density as aforementioned (8).

A number of key circumstances that might influence the FSA within the arm pull have been identified in the extant literature: (i) the head position (29), (ii) the angle of trunk inclination (23), and (iii) relative position of the upper-limb segment's (7). In the present study, swimmers were invited

TABLE 2. Comparison between the variables assessed without a FSA and swim velocity variation and with variation.

\begin{tabular}{|c|c|c|c|c|c|c|c|c|c|}
\hline & \multirow[b]{2}{*}{$\Delta, \%$} & \multirow[b]{2}{*}{ Mean Difference (95CI) } & \multicolumn{3}{|c|}{ Paired Samples $t$-Test } & \multicolumn{4}{|c|}{ Simple Linear Regression } \\
\hline & & & $t$ & $P$ & $d$ & $R^{2}$ & $\mathbf{R a}^{2}$ & SEE & $P$ \\
\hline FSA vs FSA_variation & 15.69 & $0.0142(0.0073-0.0210)$ & 4.40 & $<0.001$ & 0.95 & 0.22 & 0.17 & 0.013 & 0.055 \\
\hline$D_{\mathrm{a}}$ vs $D_{\text {a_variation }}$ & 16.09 & $13.02(5.47-20.57)$ & 3.66 & 0.002 & 0.61 & 0.76 & 0.75 & 13.01 & $<0.001$ \\
\hline$P_{\mathrm{d}}$ vs $P_{\mathrm{d} \text { _variation }}$ & 16.09 & $21.36(8.71-34.00)$ & 3.58 & 0.002 & 0.55 & 0.85 & 0.84 & 18.97 & $<0.001$ \\
\hline$P_{\text {ext }}$ vs $P_{\text {ext_variation }}$ & 16.09 & $68.88(27.56-110.19)$ & 3.53 & 0.003 & 0.53 & 0.80 & 0.78 & 71.78 & $<0.001$ \\
\hline$P_{\mathrm{k}}$ vs $P_{\mathrm{k}-\text { variation }}$ & 16.09 & $47.52(18.81-76.23)$ & 3.51 & 0.003 & 0.52 & 0.78 & 0.77 & 52.05 & $<0.001$ \\
\hline$\dot{E}_{\text {tot }}$ vs $\dot{E}_{\text {tot_variation }}$ & 16.09 & 344.38 (137.79-550.96) & 3.53 & 0.003 & 0.53 & 0.80 & 0.78 & 358.92 & $<0.001$ \\
\hline
\end{tabular}

Mean difference, mean difference in SI units; $t, t$-test; $P$, significance value; $d$, Cohen $d$ (effect size index). 

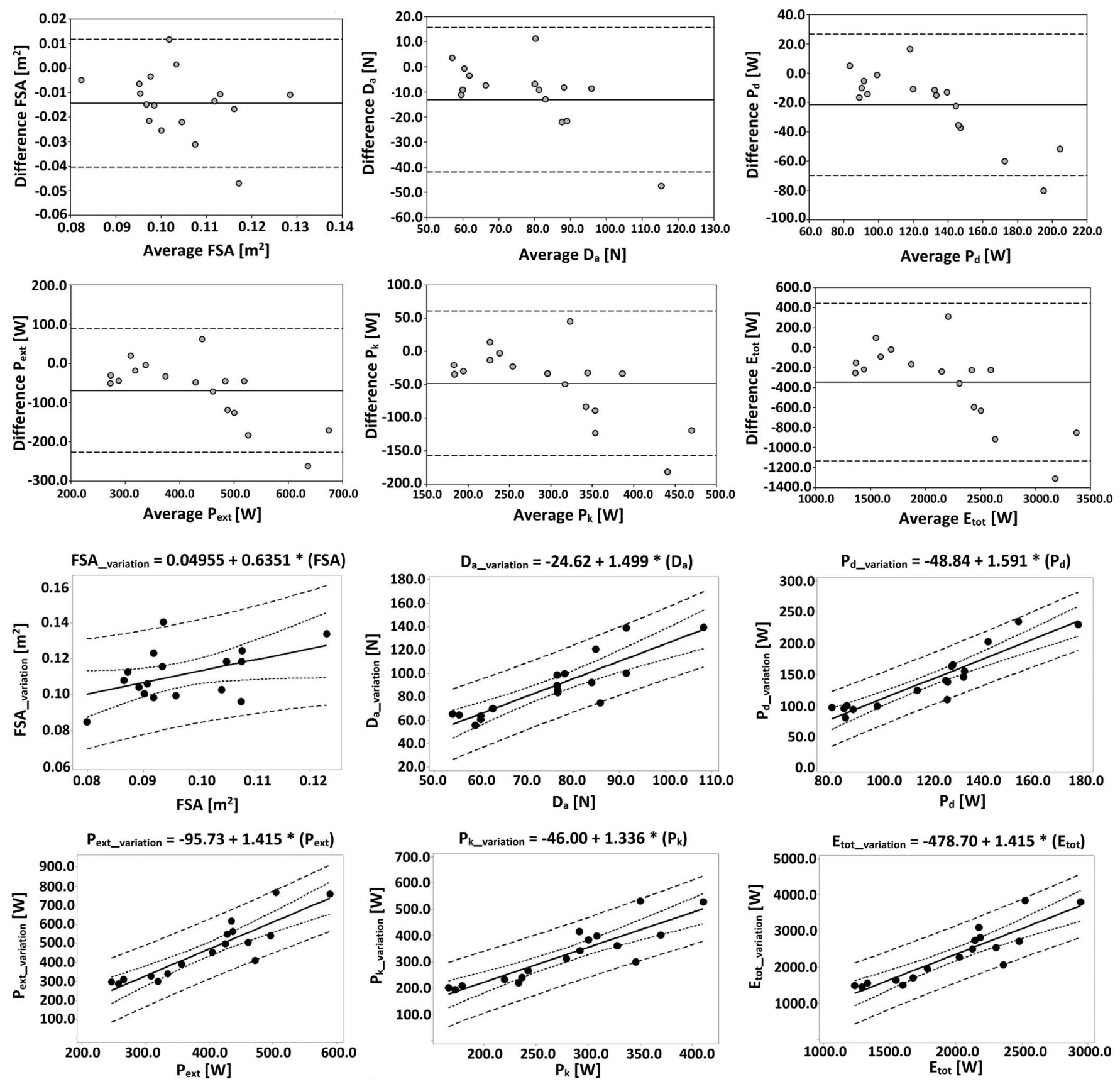

FIGURE 3-Bland-Altman and regression plots between the variables assessed without active drag variation and with active drag variation. Variation suffix, measurement performed with variation. Regression: solid line represents the trend line, small dash line represents the 95CI, long dash line represents the 95PI.

to perform nonbreathing stroke cycles during the data collection phase (between the 11th and 24th meters). This neutral position of the swimmer's head allowed him/her to minimize the effect of the head on the FSA (29). In addition, as the trials were performed at maximal velocity, one can claim that inclination of the trunk may not increase the FSA, because the small inclination of the trunk at maximum velocity does not affect the FSA (23). Therefore, the swimmers were capable of maintaining a horizontal body position at maximal swimming velocity.

The relative position of the upper limbs during the arm pull was the major factor influencing the FSA variation during the front crawl swimming (7). Indeed, in the present study, FSA was significantly higher in the variation condition than in the nonvariation one (Tables 1and 2). This was specifically noted for the key events: 2) right upper-limb insweep and 4) left upper-limb insweep. In such key events, swimmers have their hand, forearm, and upper arm out of the bounds of the trunk, leading to an FSA increase. It seems that these key events of the arm pull were the main responsible for the difference between the FSA measured in land (nonvariation) versus inwater (variation), enhancing that whenever FSA is measured, its variation should not be neglected. Consequently and based 
on this assumption, the $D_{\text {a variation }}$ also presented higher values than the nonvariation condition (Tables 1 and 2).

Indeed, it was reported that changes in the relative position of swimmers' upper limbs have a direct effect on their $D_{\mathrm{a}}$ and $C_{\mathrm{Da}}$ (i.e., a higher FSA leads to a higher $D_{\mathrm{a}}$ and $C_{\mathrm{Da}}$ ) (30). However, whenever the $D_{\mathrm{a}}$ is measured, researchers tend to use a mean swim velocity of the full stroke cycle (8). That is, swim velocity is not measured in each key event of the arm pull. This leads to measurement bias because drag is not linearly related to the swim velocity; that is, higher values of $v$ will have a larger effect on drag and vice versa (10). As during the full stroke cycle changes in FSA and $v$ are verified, these results enhance the importance of computing both the FSA and $v$ in each key event of the arm pull so that the drag measurement does not present a bias.

Assuming the $D_{\text {a variation }}$ as the "true" value for drag during the arm pull, it should be highlighted that whenever the $D_{\mathrm{a}}$ is measured without FSA and $v$ variation, an assessment bias exists. Neglecting FSA and $v$ variation led to an underestimation of the $D_{\mathrm{a}}$, mechanical power $\left(P_{\mathrm{d}}, P_{\text {ext }}\right.$, and $\left.P_{\mathrm{k}}\right)$, and $E_{\mathrm{tot}}$ (also known as energy expenditure) variables. In time-based sports, such as competitive swimming, velocity depends on the power input and energy cost of transportation:

$$
v=\frac{\dot{E}_{t o t}}{C}
$$

where $v$ is the swim velocity, $\dot{E}_{\text {tot }}$ is the total power input, and $C$ is the energy cost of swimming. $\dot{E}_{\text {tot }}$ is the sum of the contributions by the three energetic pathways:

$$
\dot{E}_{\text {tot }}=\dot{E}_{\text {aer }}+\dot{E}_{\text {anaer-la }}+\dot{E}_{\text {ATP-PCr }}
$$

where $\dot{E}_{\text {tot }}$ is the total power input, and $\dot{E}_{\text {aer }}$ is the energy contribution by the aerobic system, $\dot{E}_{\text {anaer-la }}$ by the anaerobic lactic system, and $\dot{E}_{\text {ATP-PCr }}$ by the anaerobic alactic system. Conversely, $C$ depends on the work to overcome drag force and the system's efficiency:

$$
C=\frac{w_{\mathrm{d}}}{\eta_{\mathrm{m} \eta_{\mathrm{F}}}}
$$

where $C$ is the energy cost, $w_{\mathrm{d}}$ is the mechanical work to overcome drag, $\eta_{\mathrm{m}}$ is the mechanical efficiency, and $\eta_{\mathrm{F}}$ is the Froude efficiency. Studies reported that a larger power input would be related to faster swims and also to swimmers of better competitive level $(24,25,31)$. Total power input $\left(\dot{E}_{\text {tot }}\right)$ can be estimated by Equation (8), requiring the power to overcome drag, Froude efficiency, and mechanical efficiency as inputs. Also, studies noted that less energy cost of swimming was related to faster swims or higher competitive levels $(24,31)$.

To have a better insight into the amount of power to overcome drag that a swimmer is able to produce, it is convenient to assess the external mechanical power and the mechanical power to transfer kinetic energy to water. If the latter two are large enough, one can argue that there is room to improve the power to overcome drag (i.e., by enhancing the Froude efficiency) and therefore the swim velocity. As such, the estimation of a comprehensive set of mechanical power variables enables a more holistic assessment of the potential room for improvement by the swimmer. Because the mechanical power variables and energy expenditure $\left(\dot{E}_{t o t}\right)$ are estimated based on the $D_{\mathrm{a}}$, all of them presented a significant relative difference of $16.09 \%$ (higher) when assuming an FSA, $v$, and $D_{\mathrm{a}}$ variation. Nonetheless, for all variables, a moderate-very high relationship with a high concordance was noted between assessments (Table 2, Fig. 3). Therefore, in the impossibility of assessing such variations to measure the $D_{\mathrm{a}}$ and other variables related to it, practitioners are advised to use a correction value to minimize the difference between assessments (26).

Overall, it was verified that the FSA variation during the front crawl arm pull affects $D_{\mathrm{a}}$. Therefore, one might claim that minimizing the FSA throughout the stroke cycle would lead to a decrease in $D_{\mathrm{a}}$. As depicted in Figure 1 (panels $1-5$ ), swimmers used a "propeller" movement where an elbow flexion is noted to improve the mechanical advantage of moving the hand through the water. However, this is a very controversial issue. For instance, a study by Loebbecke and Mittal (32) indicated that using a deep catch (i.e., without a meaningful flexion of the elbow during the insweep) would result in a lower FSA (due to a lower extension of the shoulder) and a higher thrust than when using the propeller technique with a shallower catch. Indeed, it was indicated that swimmers' anthropometrics and technique could explain the differences in drag (33).

Like FSA variation, the swim velocity variation also determines the $D_{\mathrm{a}}$ measurement. Studies reported that velocity varies within a cycle because of the various propulsive and recovery phases $(12,34)$. However, such studies did not indicate how such variation affects drag. The results of the present study indicate that $D_{\text {a }}$ measurement is highly dependent on FSA and swim velocity variation. Altogether, $D_{\mathrm{a}_{-} \text {variation }}$ and consequently the mechanical power variables computed based on this approach were higher than in a nonvariation approach. Despite the latter approach (i.e., nonvariation) being the most practiced (simpler and less time-consuming), researchers and practitioners should be aware of the possibility of significant differences between outcomes.

As for future research, it could highlighted that: 1) given that the propulsive phases in key events 2 and 4 (Fig. 1) represent the largest propulsion but also drag force (Table 1; Fig. 2, panel 3), researchers should investigate the effect of body and limb positions on propulsive and drag forces. Such outputs may allow coaches on giving technical cuing and feedback to manipulate posture and arm positioning. 2) It was selected a constant $C_{\mathrm{Da}}$ to model the active drag with variation. Numerical simulations can test if the $C_{\mathrm{Da}}$ changes during the full stroke cycle. Upper limbs change their position over the arm pull, and hence, it can affect the hydrodynamics. 3) One can argue that it remains to be seen if the procedures reported here are valid for other cohorts besides young elite swimmers. That said, it is not possible to foresee significant differences in the validity of this technique, as the procedure seems to be robust enough to estimate $C_{\mathrm{Da}}$ across a wide 
range of swimmers. To model FSA_variation, we have selected five standard key events of the arm pull. Besides that, the Velocity Perturbation method is selected on a regular basis to run hydrodynamic testing on different cohorts of swimmers.

As a conclusion, FSA and $v$ change during the front crawl arm pull. This variation had a significant effect on the $D_{\mathrm{a}}$, mechanical power, and total power input variable measurement. This resulted in higher values in a variation approach than a nonvariation approach because of FSA being higher in a variation condition in key events 2 and 4 . Researchers and practitioners should be aware that there is considerable bias when

\section{REFERENCES}

1. Mollendorf JC, Termin AC 2nd, Oppenheim E, Pendergast DR. Effect of swim suit design on passive drag. Med Sci Sports Exerc. 2004;36(6):1029-35.

2. Bartolomeu RF, Costa MJ, Barbosa TM. Contribution of limbs' actions to the four competitive swimming strokes: a nonlinear approach. J Sports Sci. 2018;36(16):1836-45.

3. Berger MA, Hollander AP, De Groot G. Technique and energy losses in front crawl swimming. Med Sci Sports Exerc. 1997;29(11):1491-8.

4. Vilas-Boas JP, Costa L, Fernandes RJ, et al. Determination of the drag coefficient during the first and second gliding positions of the breaststroke underwater stroke. J Appl Biomech. 2010;26(3):324-31.

5. Morais JE, Costa MJ, Mejias EJ, Marinho DA, Silva AJ, Barbosa TM. Morphometric study for estimation and validation of trunk transverse surface area to assess human drag force on water. J Hum Kinet. 2011;28:5-13.

6. Taiar R, Lodini A, Rouard A, Toshev Y. Estimation of swimmers anthropometric parameters and surface areas in real swimming conditions. Acta Bioeng Biomech. 2005;7(1):85-95.

7. Gatta G, Cortesi M, Fantozzi S, Zamparo P. Planimetric frontal area in the four swimming strokes: implications for drag, energetics and speed. Hum Mov Sci. 2015;39:41-54.

8. Kjendlie PL, Stallman RK. Drag characteristics of competitive swimming children and adults. $J$ Appl Biomech. 2008;24:35-42.

9. Pendergast D, Mollendorf J, Zamparo P, Termin A, Bushnell D, Paschke D. The influence of drag on human locomotion in water. Undersea Hyperb Med. 2005;32(1):45-57.

10. Toussaint HM, Roos PE, Kolmogorov S. The determination of drag in front crawl swimming. J Biomech. 2004;37(11):1655-63.

11. Seifert L, Chollet D, Chatard JC. Kinematic changes during a 100-m front crawl: effects of performance level and gender. Med Sci Sports Exerc. 2007;39(10):1784-93.

12. Psycharakis SG, Naemi R, Connaboy C, McCabe C, Sanders RH. Three-dimensional analysis of intracycle velocity fluctuations in front crawl swimming. Scand J Med Sci Sports. 2010;20(1):128-35.

13. Psycharakis SG, Sanders RH. Shoulder and hip roll changes during 200-m front crawl swimming. Med Sci Sports Exerc. 2008;40(12): 2129-36.

14. Nikodelis T, Kollias I, Hatzitaki V. Bilateral inter-arm coordination in freestyle swimming: effect of skill level and swimming speed. J Sports Sci. 2005;23(7):737-45.

15. Barbosa TM, Bartolomeu R, Morais JE, Costa MJ. Skillful swimming in age-group is determined by anthropometrics, biomechanics and energetics. Front Physiol. 2019;10:73.

16. Toussaint HM, Knops W, De Groot G, Hollander AP. The mechanical efficiency of front crawl swimming. Med Sci Sports Exerc. 1990; 22(3):402-8.

17. Morais JE, Jesus S, Lopes V, et al. Linking selected kinematic, anthropometric and hydrodynamic variables to young swimmer performance. Pediatr Exerc Sci. 2012;24(4):649-64. measuring such variables assuming a nonvariation of the FSA and $v$. Moreover, it should be highlighted that the variation of FSA throughout the stroke cycle should be considered when assessing active drag.

This work is supported by national funds (FCT-Portuguese Foundation for Science and Technology) under the project UIDB/04045/ 2020. There were no conflicts of interests.

The results of this study do not constitute endorsement by the American College of Sports Medicine. The results of the study are presented clearly, honestly, and without fabrication, falsification, or inappropriate data manipulation.

18. Kolmogorov SV, Duplishcheva OA. Active drag, useful mechanical power output and hydrodynamic force coefficient in different swimming strokes at maximal velocity. J Biomech. 1992;25(3):311-8.

19. Marinho DA, Barbosa TM, Costa MJ, et al. Can 8-weeks of training affect active drag in young swimmers? J Sports Sci Med. 2010; 9(1):71-8.

20. Morais JE, Silva AJ, Marinho DA, Seifert L, Barbosa TM. Cluster stability as a new method to assess changes in performance and its determinant factors over a season in young swimmers. Int $J$ Sports Physiol Perform. 2015;10(2):261-8.

21. Neiva HP, Marques MC, Barbosa TM, Izquierdo M, Marinho DA. Warm-up and performance in competitive swimming. Sports Med. 2014;44(3):319-30.

22. Zamparo P. Effects of age and gender on the propelling efficiency of the arm stroke. Eur J Appl Physiol. 2006;97:52-8.

23. Zamparo $P$, Gatta G, Pendergast D, Capelli C. Active and passive drag: the role of trunk incline. Eur J Appl Physiol. 2009;106(2):195-205.

24. Zamparo P, Pendergast DR, Mollendorf J, Termin A, Minetti AE. An energy balance of front crawl. Eur J Appl Physiol. 2005;94:134-44.

25. Pendergast D, Zamparo P, Di Prampero PE, et al. Energy balance of human locomotion in water. Eur J Appl Physiol. 2003;90(3-4):377-86.

26. Barbosa TM, Ramos R, Silva AJ, Marinho DA. Assessment of passive drag in swimming by numerical simulation and analytical procedure. J Sports Sci. 2018;36(5):492-8.

27. Cohen J. Statistical Power Analysis for the Behavioral Sciences. 2nd ed. Hillsdale (NJ): Lawrence Erlbaum Associates; 1988. p. 567.

28. Bland JM, Altman DG. Statistical methods for assessing agreement between two methods of clinical measurement. Lancet. 1986;1(8476):307-10.

29. Zaidi H, Taiar R, Fohanno S, Polidori G. Analysis of the effect of swimmer's head position on swimming performance using computational fluid dynamics. $J$ Biomech. 2008;41(6):1350-8.

30. Formosa D, Mason BR, Burkett BJ. Measuring active drag within the different phases of front crawl swimming. In: Proceedings of the XIth International Symposium for Biomechanics and Medicine in Swimming. Oslo, Norway: Norwegian School of Sport Sciences; 2010. pp. 82-4.

31. Barbosa TM, Bragada JA, Reis VM, Marinho DA, Carvalho C, Silva AJ. Energetics and biomechanics as determining factors of swimming performance: updating the state of the art. J Sci Med Sport. 2010;13(2):262-9.

32. Loebbecke AV, Mittal R. Comparative analysis of thrust production for distinct arm-pull styles in competitive swimming. J Biomech Eng. 2012;134(7):074501.

33. Narita K, Nakashima M, Takagi H. Developing a methodology for estimating the drag in front-crawl swimming at various velocities. J Biomech. 2017;54:123-8.

34. Barbosa TM, Morouço PGF, Jesus S, et al. The interaction between intracyclic variation of the velocity and mean swimming velocity in young competitive swimmers. Int J Sports Med. 2013;34(2):123-30. 